\title{
Diagnóstico de sospecha de cáncer en los servicios de urgencias
}

\author{
A. Darias Acosta ${ }^{1}$, C. Gironés Bredy ${ }^{1,2}$, M. Benito Lozano ${ }^{1}$, G. Burillo-Putze ${ }^{1,2}$
}

\section{Sr. Director:}

El trabajo de Lana y col publicado recientemente en su revista presenta unos resultados sorprendentes y da pie a algunas reflexiones ${ }^{1}$.

En primer lugar, a tenor de los datos expuestos parece necesario revisar el funcionamiento de la Atención Primaria asturiana en la que a diagnóstico precoz del cáncer se refiere, al ser los casos diagnosticados en los servicios de Urgencias Hospitalarias (SUH) -10,4\%- superiores a los que diagnostica la medicina familiar y comunitaria $(6,8 \%)$ y, lo que resulta más preocupante, con un diagnóstico de estadio avanzado en el momento del diagnóstico en los SUH en el $33 \%$ de los $\operatorname{casos}^{1}$. Ciertamente no conocemos lo que ocurre en otras comunidades autónomas españolas.

Además, si el paciente con sospecha de cáncer entra en el sistema sanitario a través de los $\mathrm{SUH}$, se reduce el tiempo en que se confirma el diagnóstico de sospecha y el tiempo en que se inicia el tratamiento, en 10 y 15 días respectivamente ${ }^{1}$. Con estos dos datos, no es de extrañar que un paciente con preocupación por su salud, y concretamente con la posibilidad de padecer una neoplasia acuda directamente a los SUH, con la consiguiente saturación de estos servicios ${ }^{2}$. Como afirmaba Sesma en esta revista, los SUH han ido paulatinamente conformando la "red de seguridad

1. Servicio de Urgencias. Hospital Universitario de Canarias. Tenerife. España

2. Unidad de Farmacología. Departamento de Medicina Física y Farmacología. Universidad de La Laguna. Tenerife. España asistencial" del Sistema Nacional de Salud (SNS), soportando las debilidades del mismo, el cual se inhibe de ellas a sabiendas de que los SUH "resuelven muchas de las disfunciones del día a día que el propio sistema genera"

Es lógico que un paciente con síntomas de alarma (hemoptisis, rectorragia, metrorragia, hematuria, focalidad neurológica, etc) acuda al servicio de Urgencias ${ }^{4}$, también es lógico que los SUH demos soporte a las complicaciones agudas de los tratamientos oncológicos (sobre todo en los horarios en que no funcionan los hospitales de día oncológicos $)^{5}$, pero no nos parece lógico incorporar a la rutina de los $\mathrm{SUH}$ algoritmos de diagnóstico de presunción de cánceres por sus beneficios para el sistema y para los pacientes ${ }^{1}$. Como la experiencia nos dice que asumir más funciones asistenciales en los SUH no va siempre unido a un aumento de espacios, recursos y, por qué no decirlo, reconocimiento, deben potenciarse como también apuntan los autores, las unidades de diagnóstico rápido ${ }^{6}$ y la mejor coordinación entre atención primaria y atención hospitalaria?

Mención aparte merecen los cuidados paliativos en los SUH que reciben en ocasiones estos pacientes, los cuales creemos que no deberían compartir espacios y dinámicas asistenciales con los $\mathrm{SUH}$, tanto desde el punto de vista del paciente como de su entorno.

\section{Correspondencia:}

Dr. Guillermo Burillo Putze

Servicio de Urgencias

Hospital Universitario de Canarias

C/ Ofra s/n

38320 La Laguna. Tenerife.

E-mail: gburillo@telefonica.net 
Acostumbrados como estamos a suplir las carencias del SNS y a conocer sus puntos negros, parece lógico que en determinadas estrategias nacionales de salud, se cuente con la medicina de urgencias, en ocasiones infrarrepresentada cuando no ausente. Los datos tristemente así lo indi$\mathrm{can}^{8}$.

\section{BIBLIOGRAFÍA}

1. Lana A, Álvarez-Guerrero S, Herrero-Puente P, Folgueras M, López M. Diagnóstico de sospecha del cáncer en los servicios de urgencia hospitalario. An Sist Sanit Navar 2014; 37: 59-67.

2. Miró O, Escalada X, Boqué C, Gené E, Jiménez FáBREGA FX, NETTO C et al. Estudio SUHCAT (2): mapa funcional de los servicios de urgencias hospitalarios de Cataluña. Emergencias 2014; 26: 35-46.

3. Sesma SÁnchez J. Saturación en los servicios de urgencias hospitalarios. An Sist Sanit Navar 2012; 35: 195-198.
4. Gómez-Zorrilla Martín S, Riera-Mestre A, Leiva Pedraza D, Jordán lucas S, Jacob Rodríguez J, PuJol FarRIOLS R. Diagnóstico alternativo al tromboembolismo pulmonar en urgencias en pacientes oncológicos y no oncológicos. Emergencias 2013; 25: 92-98.

5. Sadik M, Ozlem K, Huseyin M, AliAyberk B, AHMET S, OzGUR O. Attributes of cancer patients admitted to the emergency department in one year. World J Emerg Med 2014; 5: 85-90.

6. M. de Miguel Valencia M, Esquíroz Lizaur I, Marzo Virto J, de Miguel Velasco M, Yagüe HernanDo A. Invaginación intestinal como primera manifestación del cáncer de pulmón. An Sist Sanit Navar 2014; 37: 299-304.

7. Mirpuri-Mirpuri P, Álvarez-Cordovés MM, PérezMonje A. Programa de diagnóstico precoz de cáncer colorrectal en Canarias. A propósito de un caso. Semergen 2013; 39: e71-e74.

8. Estrategia en Cáncer del Sistema Nacional de Salud. MINISTERIO DE SANIDAD Y POLÍTICA SOCIAL. Disponible en URL: http://www. msssi.gob.es/organizacion/sns/planCalidadSNS/pdf/ActualizacionEstrategiaCancer. pdf. Acceso 20/09/2014. 